\title{
Papers
}

\section{Small cell malignant melanoma: a variant of naevoid melanoma. Clinicopathological features and histological differential diagnosis}

\author{
K Blessing, J J H Grant, D S A Sanders, M M Kennedy, A Husain, P Coburn
}

Department of

Pathology, Aberdeen

University,

Foresterhill, Aberdeen

AB25 2ZD, UK

K Blessing

Department of
Pathology,
Birmingham
University,
Birmingham B15 2TT,
UK
D S A Sanders

Department of Pathology, Heartlands Hospital, Bordesly

Green East, Birmingham B9 5SS, UK

M M Kennedy

Department of Pathology, Middlesborough General Hospital, Cleveland TS5 5AZ, UK

A Husain

Departments of Pathology and Dermatology, Worthing Hospital, Sussex, BN11 2DH UK J J H Grant

Correspondence to: Dr Blessing email: k.blessing@abdn.ac.uk

Accepted for publication 13 March 2000

Table 1 Malignant melanomas that mimic benign melanocytic lesions (naevoid melanomas)

Spitzoid melanoma Desmoplastic melanoma Regressed melanoma Melanoma in stasis skin

Verrucous keratotic melanoma

Small cell melanoma

\section{Abstract}

Aims-To describe the clinical and histopathological features of a rare variant of naevoid melanoma, small cell melanoma, and discuss the histological differential diagnoses.

Methods-The clinical and histological features of cases of malignant melanoma with the histological features of small (non-Merkel like) melanoma were reviewed and documented. In addition, five cases had available material for immunohistochemistry and this was performed using antibodies to the $S 100$ protein and melan-A, and the HMB-45 antibody.

Results-There were 15 cases of small cell melanoma from 14 (10 female, four male) patients, aged between 30 and 77 (mean, 48.6) years. The trunk was the most common location. In more than half the cases, the provisional diagnosis was melanomal borderline lesion. All shared similar histological appearances of an intraepidermal component of in situ melanoma and a dermal component of nests of cells with hyperchromatic nuclei and scanty cytoplasm, usually in tightly packed nests. All components (junctional and intradermal) of the lesions investigated by immunohistochemistry were positive both for $\mathrm{S} 100$ protein and melan-A. All junctional components were positive with HMB-45, but with variable staining of the dermal components with this antibody.

Conclusions-Small cell malignant melanoma is postulated to be a distinct histopathological entity and a rare variant of naevoid melanoma. Such lesions can be difficult to interpret and easily missed at scanning magnification because the cells of the dermal component mimic benign naevus cells.

(F Clin Pathol 2000;53:591-595)

Keywords: malignant melanoma; naevoid melanoma; small cell melanoma

Malignant melanoma is renowned for its heterogeneous histological appearance, including mimicking other malignant tumours and benign melanocytic lesions. Those malignant melanomas that mimic benign melanocytic lesions are now recognised as uncommon variants that one should be aware of overlooking in daily practice (table 1).$^{1-12}$ Most could be termed naevoid (naevocytic) melanomas. They are uncommon and pose difficulties in diagnosis. We would like to add another rare variant of naevoid melanoma, called small cell melanoma, where the constituent cells are small melanoma cells. These cells resemble those frequently seen at the base of conventional melanoma. There have been several references to small cell melanoma but almost all refer to a highly malignant tumour composed of small, undifferentiated cells with a high mitotic rate, and mostly occurring in non-cutaneous sites. These small cell melanomas are Merkel cell like. ${ }^{13-16}$ There is, however, a small cell melanoma variant that is poorly described in the literature and which like the other variants listed in table 1 could easily be misdiagnosed either as a benign naevus or in situ melanoma. ${ }^{17}$ Our study describes the clinical and histological features of this entity and discusses its relation to naevoid and minimal deviation melanoma, and other potential differential diagnoses.

Patients and methods

The cases were from personal collections, including referred cases, of two authors (KB and DSAS). The criteria (table 2) were discussed with all the histopathologists in the study and based on the published criteria used in discriminating small melanoma cells and naevus cells in conventional melanomas (fig 1). ${ }^{18-20}$ The haematoxylin and eosin stained sections were available for review in all cases. In addition, the blocks were available in five cases for immunohistochemical staining with antibodies to the $S 100$ protein (Dako; 1/350 dilution) and melan-A

Table 2 Histological criteria for the diagnosis of small cell melanoma

(1) An obvious intraepidermal component recognisable at low power as in situ melanoma

(2) An intradermal component composed entirely of small dark cells mimicking benign naevus cells, usually lying in tightly packed nests

(3) The dermal nests composed of cells with hyperchromatic, slightly pleomorphic nuclei and scanty cytoplasm. There may be occasional prominent nucleoli

(4) A stromal response of proliferation of small blood vessels between the dermal nests and a variable lymphocytic infiltrate 


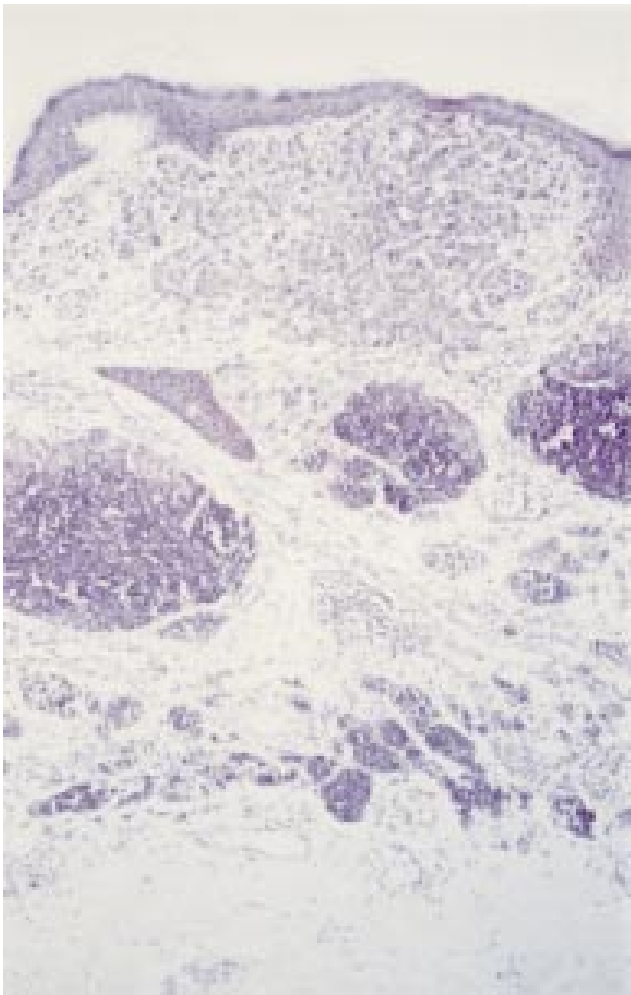

Figure 1 Conventional malignant melanoma. The cells become smaller deep in the lesion, at the base (small melanoma cells), mimicking benign naevus.

(Novocastra; 1/10 dilution), and the HMB-45 antibody (Dako; 1/50 dilution). Anti-melan-A and HMB-45 required antigen retrieval by high temperature unmasking.

The clinical features documented were patient age, patient sex, anatomical location of the lesion, lesion size, and follow up. These were obtained from request forms and case notes where possible. Histologically, the Breslow depth, Clark level, histogenetic type, pre-existing melanocytic lesion, mitotic count, lymphocytic infiltrate (scored as,,+++ , and +++ ), and vascular response (scored as,+++ , and +++ ) were assessed.

\section{Results}

There were 15 lesions identified from 14 (10 female and four male) patients, aged between 30 and 77 (mean, 48.6) years. Eight patients were aged 40 years or over, of whom six were

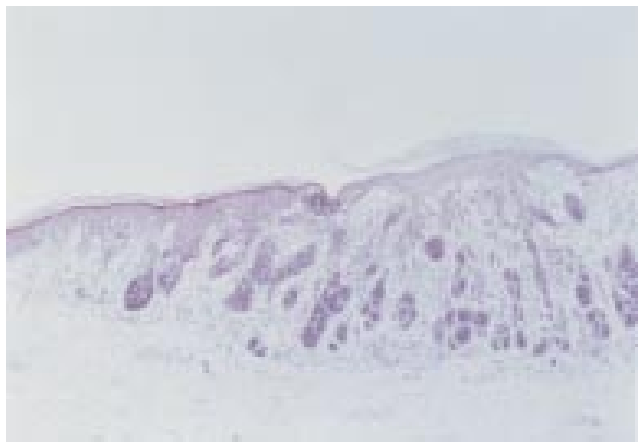

Figure 2 Small cell melanoma. Lesion from the calf of a 46 year old woman, with the clinical history of probable melanoma. Cells within the dermis are hyperchromatic and nested.

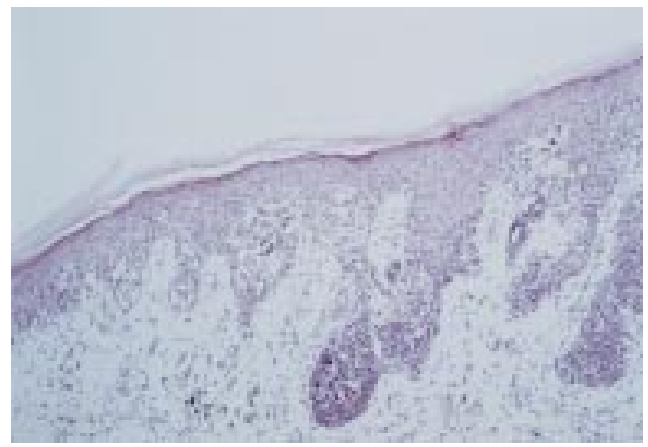

Figure 3 Small cell melanoma, as in fig 2. Note the destructive junctional component and the malignant epidermal component merging with the dermal nests.

aged 50 years or over. Seven lesions were from the trunk, three from the upper limb, and five from the lower limb. No lesion was from an acral or genital site. All lesions were cutaneous in origin. The lesions measured 4-12 (mean, 7.0) $\mathrm{mm}$. One patient was described as having "multiple moles", one patient clinically had the atypical mole syndrome (dysplastic naevus syndrome), and another patient developed two lesions at different sites and on separate occasions. These last two lesions were felt not to be metastases and to be two independent lesions. Four lesions were submitted as probable melanomas, three with a history of borderline lesion/melanoma, two as probable dysplastic naevi, two as dysplastic naevi/other, two were accompanied with a history of mole with recent change, and two lesions had no accompanying history.

Initially, three lesions had been called benign naevi (one lentiginous compound naevus, one compound naevus with atypia, and one just naevus); another one had been reported as in situ melanoma. On review, all were regarded as malignant melanoma (of the small cell type). The intraepidermal component was in situ melanoma either recapitulating superficial spreading melanoma or having a lentiginous pattern in non-atrophic skin (figs 2-5). On high power examination, the dermal nests were composed of cells with hyperchromatic nuclei, exhibiting mild to moderate nuclear pleomorphism, and scanty cytoplasm. These nests lacked maturation at the base of the lesion. Many of the nests showed an expansile growth pattern, with the outer rim of nuclei being

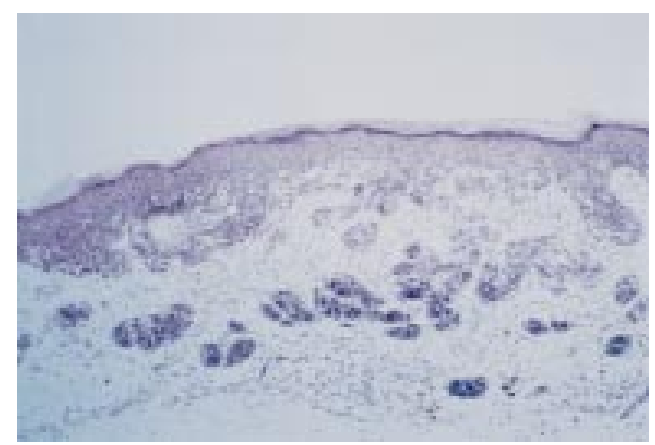

Figure 4 Small cell melanoma. Lesion from the thigh of a 30 year old woman with a clinical history of probable melanoma. Note the atypical junctional component and the merging of the epidermal and dermal components. 


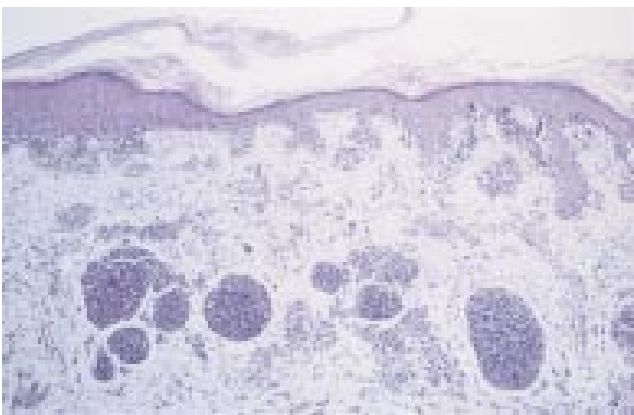

Figure 5 Small cell melanoma. Lesion from the thigh of a 56 year old woman. No clinical history was supplied. There is a lentiginous proliferation of melanocytes at the junction. There is a dermal component composed of expansile nests of small cells with hyperchromatic nuclei.

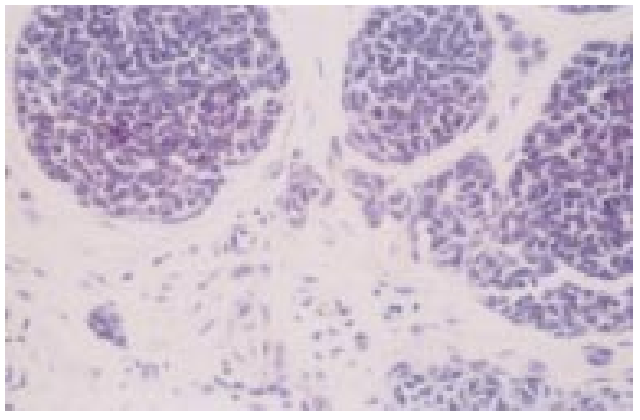

Figure 6 High power of the dermal nests as in fig 5. The nests have flattened peripheral nuclei, suggesting expansile growth. The constituent cells have scanty cytoplasm, mild nuclear pleomorphism, and occasional nucleoli.

flattened and spindle shaped (fig 6). In two cases, there was mergence of the intraepidermal component and the dermal component (figs 2-4). Between the nests there was vascular proliferation and a lymphocytic infiltrate supporting a malignant diagnosis (fig 6).

All were classified as melanomas with small melanoma cell morphology, with the Breslow depth ranging from 0.4 to 1.1 (mean, $0.63) \mathrm{mm}$. Only one lesion, however, measured over $1.0 \mathrm{~mm}(1.1 \mathrm{~mm})$. Five lesions reached Clark level IV, the remainder reaching level III. Eight lesions had a lymphocytic infiltrate graded as $2+$, seven with an infiltrate grade of $1+$. The vascular response between the tumour islands was graded as $2+$ in 11 patients and $1+$ in four. Mitotic figures were not identified in the dermal component of any lesion. Follow up has ranged from four to 96 (mean, 36.2) months. No patient has developed recurrence or metastasis, but as noted earlier, in only one patient was the lesion over $1.0 \mathrm{~mm}(1.1 \mathrm{~mm})$, and this patient has been followed up for about 12 months only. The patient with the two melanomas has had several basal cell carcinomas removed, and the patient with multiple moles and another with atypical mole syndrome have had other melanocytic lesions removed that were felt to be benign but showed mild architectural atypia.

All components of all available lesions showed diffuse staining for $\mathrm{S} 100$ protein and melan-A. The junctional components of all the specimens were diffusely positive with $\mathrm{HMB}-45$, as well as the dermal components in one lesion. The other samples showed variable patchy positivity of the dermal components with HMB-45.

\section{Discussion}

There is increasing recognition of melanoma variants and we believe that small cell melanoma is an important, but uncommon, variant that could easily be dismissed as a benign naevus or an in situ melanoma because the dermal nests can mimic a benign naevus at scanning power. Unlike other naevoid melanomas, the constituent cells are small melanoma cells that are frequently seen at the base of otherwise conventional melanomas. This has been called apparent maturation ${ }^{11}$ or pseudonaevoid phenomenon, ${ }^{5}$ and describes the melanoma cells deep within a lesion that become smaller but retain nuclear atypia, hyperchromasia, prominent nucleoli, and a large nuclear to cytoplasmic ratio. ${ }^{11}$ These areas within conventional melanomas can cause problems of differentiating pre-existing naevus cells from small melanoma cells. Features cited as favouring melanoma include cells with hyperchromatic nuclei, prominent nucleoli, nuclei more than $10 \mu \mathrm{m}$ in diameter, newly formed blood vessels, a lymphocytic infiltrate, and pronounced fibrosis. ${ }^{18-20}$ Mitotic figures obviously favour a diagnosis of melanoma, but Maize et al state that they are seldom present. ${ }^{18}$ Elder and Murphy emphasise the need for careful evaluation of the epidermal component, and suggest that mitoses in the dermis although helpful are not necessary for the diagnosis. ${ }^{19}$

One major problem with acceptance of the concept of melanomas of the small cell type as being malignant is that the constituent cells are small, and exhibit only mild cytological abnormalities. In addition, there have been no metastases from this series. The differential diagnosis must therefore include "atypical naevi" (dysplastic naevi) or in situ melanoma with atypical dermal nests. However, the existence of the former group of lesions is also extremely contentious. ${ }^{21} 22$ As with tumours in other organs, malignancy in melanocytic lesions can only be proved if metastasis occurs and, therefore, virtually all the evidence is indirect. The first line of evidence is the clinical features: the age of the patients, the size of the lesions, and the presenting history. Naevoid melanomas are favoured in preference to naevus when the patient is 40 years or older and the lesions are $\geqslant 6 \mathrm{~mm} .{ }^{20}$ In addition, because most of the lesions were submitted with a suspicious clinical history, it would be considered unwise to interpret a junctional component with a lentiginous or pagetoid growth pattern as seen in figs $2-5$ as benign. The dermal component again is contentious, but conforms to most of the accepted published criteria for small melanoma cells. Also, the nests were frequently expansile, a feature more in keeping with melanoma than naevus. As noted earlier mitoses are not required. We agree with Maize et al that mitotic figures are rarely or almost never found in these pseudonaevoid nests at the base of conventional melanomas. ${ }^{18}$ It may be that this small cell melanoma variant has similarities to desmoplastic melanoma: that is, a distinctive dermal growth pattern, and this could be the result of epidermally derived growth factors. 
There are currently no reliable immunohistochemical techniques that readily allow the distinction between benign and malignant melanocytes. The fact, however, that some of the dermal nests stained with HMB-45 is slightly supportive of our interpretation of these lesions being malignant. ${ }^{2324}$

There have been several other descriptions of small cell melanoma, some describing sheets of small hyperchromatic cells with a high mitotic rate (Merkel cell like), usually occurring in non-cutaneous sites. ${ }^{13-16}$ In these melanomas and their metastases, immunohistochemistry was required to exclude other malignant small round cell tumours. However, we believe that the term small cell melanoma should be reserved specifically for the lesions described in our study and one other publication. ${ }^{17}$

Whether or not this and other variants of naevoid melanoma are less aggressive remains to be confirmed or refuted by larger studies. Kossard et al studied the proliferative activity and nuclear morphology in an apparently similar group of lesions with small cell morphology and compared them with conventional naevi and typical melanomas. They showed that the average numbers of argyrophilic nucleolar organiser regions in each nucleus for small cell melanoma was midway between those of benign naevi and typical melanomas. In addition, using digital image analysis, they demonstrated that the nuclear perimeter and nuclear area of the cells in small cell melanoma did not differ significantly from ordinary dermal naevus, but both groups differed significantly from conventional melanomas. ${ }^{17}$ However, they were uncertain as to whether these results indicated that small cell melanoma had a better prognosis than conventional melanomas. They also did not comment on the stage of their samples, and one was $2.3 \mathrm{~mm}$ and could have metastasised. One report describes metastasis of small cell melanoma to the stomach, but whether or not the primary lesion fulfilled the criteria for small cell melanoma used in the study of Kossard et al and our study is unclear. $^{14}$

There is confusion over the use of the term "naevoid" and in some instances minimal deviation melanoma to describe this group of small cell melanoma. Naevoid means naevus like, and therefore it is a general term and could be used to describe all the variants of melanoma listed in table 1 . Some of these variants have distinct histological features, but some of the reports of naevoid melanoma contain a heterogeneous group of lesions. ${ }^{6-12}$ Some have the cytological features of conventional melanomas (verrucous naevoid), whereas some have an intermediate cytology ("dome shaped lesions" described by Wong et al). ${ }^{11} \mathrm{It}$ might be that the small cell melanoma is a subgroup of these dome shaped naevoid melanomas, with the constituent cells being small melanoma cells. Therefore, it is important to group only similar lesions together to assist in assessing prognostic implications and to enable comparisons of studies to be carried out.

Some authors believe that some of the "naevoid" melanomas listed in table 1 might also be called minimal deviation or borderline melanoma. These terms are used to describe a subgroup of melanomas thought to have a less aggressive clinical course and a vertical growth phase, which have been described as having less pronounced cytological atypia than conventional melanomas. ${ }^{25-27}$ The terminology has proved difficult and confusing for most practising pathologists to grasp. The principal problems are the lack of clearly defined criteria for making the diagnosis, with the result that it may encompass a heterogeneous group with all types of lesions, possibly including benign naevi. This makes it difficult to interpret prognostic studies. Describing multiple subtypes of this minimal deviation melanoma ${ }^{27}$ has further confused the concept. In addition, whereas minimal deviation melanomas are level IV by definition, the term borderline melanomas is given to similar lesions that reach only level III. Increasingly, dermatologists and pathologists are using the term borderline lesion to describe a pigmented lesion that is severely atypical and bordering on in situ melanoma. For these reasons, although small cell melanoma might have a less aggressive behaviour, we also believe that the terms minimal deviation and small cell melanoma are not interchangeable.

In conclusion, the term small cell melanoma should be used to describe an uncommon variant of melanoma that belongs to the larger family of naevoid melanomas, all of which can mimic benign melanocytic lesions. This variant of melanoma might have a better prognosis than conventional melanomas. These lesions should be distinguished from the Merkel like small cell melanoma, which is predominantly of non-cutaneous sites. Larger studies, including analysis of growth factor production, with further assessment using newer proliferation markers, are required to confirm or refute the malignant nature of this group of lesions, and to determine the prognostic importance of making this diagnosis.

1 Spitz S. Melanomas of childhood. Am F Pathol 1948;24:591609.

2 Smith KT, Skelton HG, Lupton GP, et al. Spindle cell and epithelioid cell nevi with atypia and metastasis (malignant Spitz nevus). Am $\mathcal{F}$ Surg Pathol 1989;13:931-9.

3 Jain S, Allen PW. Desmoplastic malignant melanoma and its variants. A study of 45 cases. Am $\mathcal{f}$ Surg Pathol variants. A stucy

4 Blessing K, McLaren KM. Histological regression in primary cutaneous melanoma: recognition, prevalence and significance. Histopathology 1992;20:315-22.

5 Blessing K. Malignant melanoma in stasis dermatitis. Histopathology 1997;30:135-9.

6 Levene A. On the histological diagnosis and prognosis of malignant melanoma. F Clin Pathol 1980;33:101-24.

7 Suster S, Ronnen M, Bubis JJ. Verrucous pseudonaevoid melanoma. F Surg Oncol 1987;36:134-7.

8 Kuehnl-Petzoldt C, Berger H, Wiebelt H. Verrucouskeratotic variations of malignant melanoma. Am f Dermatopathol 1982;4:403-10.

9 Schmoeckel C, Castro CE, Braun-Falco O. Nevoid malignant melanoma. Arch Dermatol Res 1985;277:362-9. 
10 Blessing K, Evans AT, Al-Nafussi A. Verrucous naevoid and keratotic malignant melanoma: a clinicopathological study of 20 cases. Histopathology 1993;23:453-8.

11 Wong TY, Suster S, Duncan LM, et al. Nevoid melanoma. Hum Pathol 1995;26:171-9.

12 McNutt NS. "Triggered trap": nevoid malignant melanoma. Semin Diagn Pathol 1998:15:203-9.

13 House NS, Fedok F, Maloney ME, et al. Malignant melanoma with the clinical and histologic features of Merkel cell carcinoma. $\mathcal{F}$ Am Acad Dermatol 1994;31:839-42.

14 Attanoos R, Griffiths DFR. Metastatic small cell melanoma to the stomach mimicking primary gastric lymphoma. Histopathology 1992;21:173-5.

15 Barnhill RL. Childhood melanoma. Semin Diagn Pathol 1998;15:189-94.

16 Nakleh RE, Wick MR, Rocamora A, et al. Morphological diversity in malignant melanomas. Am $f$ Clin Patho diversity in malign

17 Kossard S, Wilkinson B. Nucleolar organiser regions and image analysis nuclear morphometry of small cell (nevoid) melanoma. ₹ Cutan Pathol 1995;22:132-6.

18 Maize JC, Burgdorf WHC, Hurt MA, et al. Cutaneous pathology. Edinburgh: Churchill Livingstone, 1998.

19 Elder DE, Murphy GF. Atlas of tumor pathology. Melanocytic tumors of the skin. Washington DC: Armed Forces Institute of Pathology, 1991
20 Cochrane AJ, Bailly C, Paul E, et al. Melanocytic tumors: a guide to diagnosis. Biopsy interpretation series. Philadelphia:

21 Ackerman AB. What nevus is dysplastic, a syndrome and the commonest precursor to malignant melanoma? A riddle and an answer. Histopathology 1988;13:241-56.

22 Clark WH, Ackerman AB. An exchange of views regarding the dysplastic naevus controversy. Semin Dermatol 1989;8: $229-50$

23 Blessing K, Sanders DSA, Grant JJH. Comparison of immunohistochemical staining of the novel antibody melan-A with S100 protein and HMB-45 in malignant melanoma and melanoma variants. Histopathology 1998;32: 139-46.

24 Evans ME, Evans AT, King G, et al. Histopathological and immunohistochemical discrimination of pre-existing naevus cells from malignant melanoma [abstract]. F Pathol 1996;178:43a.

25 Reed RJ, Ichinose H, Clark WH, Jr, et al. Common and uncommon melanocytic nevi and borderline melanomas. Semin Oncol 1975;2:119-47.

26 Phillips ME, Margolis RJ, Merot Y, et al. The spectrum of minimal deviation melanoma: a clinicopathologic study of 21 cases. Hum Pathol 1986;17:796-806.

27 Reed RJ, Martin P. Variants of melanoma. Semin Cutan Med Surg 1997;16:137-58. 